Supporting Information

\title{
Quantum confined Tomonaga-Luttinger liquid in Mo6Se6 nanowires converted from epitaxial MoSe2 monolayer
}

Yipu Xia, ${ }^{\dagger,+}$ Bo Wang, ${ }^{\|,+}$Junqiu Zhang, ${ }^{\dagger,+}$ Yuanjun Jin, ${ }^{\dagger}$, Ho Tian, ${ }^{\dagger}, \S$ Wingkin Ho, ${ }^{\dagger} H u$ Xu, ${ }^{\S}$ Chuanhong Jin", and Maohai Xie ${ }^{\dagger, *}$

†Physics Department, the University of Hong Kong, Pokfulam Road, Hong Kong

"State Key Laboratory of Silicon Materials, School of Materials and Engineering, Zhejiang University, Hangzhou, Zhejiang, 310027, China

${ }^{\S}$ Department of Physics, Southern University of Science and Technology, Shenzhen, Guangdong 518055, China

These authors contributed equally to this paper.

*Corresponding authors, Emails: chhjin@zju.edu.cn (CHJ), mhxie@hku.hk (MHX).

KEYWORDS: One-dimensional system, Nanowire, Tomonaga-Luttinger liquid, MBE, STM 


\section{Formation processes of $\mathrm{Mo}_{6} \mathrm{Se}_{6}$ nanowires}

Figures S1a-c present a set of STM images revealing the formation process of $\mathrm{Mo}_{6} \mathrm{Se}_{6}$ nanowires by increasing the annealing time but at a fixed temperature of $\sim 800^{\circ} \mathrm{C}$. The $\mathrm{MoSe}_{2}$ film was pre-grown by MBE on the SiC-supported graphene substrate. As seen, at the early stage of annealing (Figure S1a), short wires emerge at the boundaries of $\mathrm{MoSe}_{2}$ islands or domains. They lengthen with increasing annealing time (Figure $\mathrm{S} 1 \mathrm{~b}$ ) and eventually the whole edge of ML $\mathrm{MoSe}_{2}$ island or domain becomes converted into $\mathrm{Mo}_{6} \mathrm{Se}_{6}$ (Figure S1c). Combining atomic resolution STM (e.g., Figure S1c inset) and annular dark-field (ADF) STEM (Figures S1d-f), we establish the atomic structure of the nanowires to be $\mathrm{Mo}_{6} \mathrm{Se}_{6}$, which is as sketched in Figure $1 \mathrm{~b}$ in the main text. In most cases, individual $\mathrm{Mo}_{6} \mathrm{Se}_{6}$ nanowires are seen at $\mathrm{MoSe}_{2}$ island or domain boundaries. In other cases, especially those under prolonged and high-temperature annealing, double, triple (Figure S1e) and even bundles (Figure S1f) of $\mathrm{Mo}_{6} \mathrm{Se}_{6}$ nanowires could form by completely dissolving a $\mathrm{MoSe}_{2}$ patch or island. The $\mathrm{Mo}_{6} \mathrm{Se}_{6}$ wires in the bundle lie parallel on substrate surface. Fourier transform (FT) of Figure S1f shows, besides spots corresponding to $\mathrm{MoSe}_{2}$ lattices, features of the $\mathrm{Mo}_{6} \mathrm{Se}_{6}$ bundle stacked parallel with an interwire distance of $\sim 0.82 \mathrm{~nm}$ (blue circles in the inset). From the diffraction spots marked by the red circles, we further derive that the lattice constant of $\mathrm{Mo}_{6} \mathrm{Se}_{6}$ along its length is $\sim 0.45 \mathrm{~nm}$ (note that the FT of a top-view image of $\mathrm{Mo}_{6} \mathrm{Se}_{6}$ reflects half of the unit cell size), consistent with our density-functional theory (DFT) calculations of the lattice constants. From Figure 1c in the main text, one may also note that some $\mathrm{Mo}_{6} \mathrm{Se}_{6}$ nanowires have formed even at the edges of the second layer $\mathrm{MoSe}_{2}$, suggesting that $\mathrm{Mo}_{6} \mathrm{Se}_{6}$ wire formation out of $\mathrm{MoSe}_{2}$ is not much associated with the identity of the substrate or the lattice registry of $\mathrm{MoSe}_{2}$ with respect to the substrate. Once formed, these $\mathrm{Mo}_{6} \mathrm{Se}_{6}$ nanowires are very stable, which can withstand both hightemperature annealing (up to $\sim 950^{\circ} \mathrm{C}$ ) and exposure to ambient for more than one week.

By annealing the $\mathrm{MoSe}_{2}-\mathrm{Mo}_{6} \mathrm{Se}_{6}$ sample under a flux of Se at temperature $\sim 800^{\circ} \mathrm{C}$, the $\mathrm{Mo}_{6} \mathrm{Se}_{6}$ nanowires will dissolve (Figure S1g). In addition, some second or higher layer $\mathrm{MoSe}_{2}$ appear at the edges of ML-MoSe 2 domain or islands (Figure S1h). Different from direct epitaxial growth of $\mathrm{MoSe}_{2}$, where the higher layers usually nucleate at the domain centers, the new $\mathrm{MoSe}_{2}$ islands formed by selenization are close to the edges of ML-MoSe 2 domains, which are thus likely caused by the dissolving or reorganizing the $1 \mathrm{D} \mathrm{Mo}_{6} \mathrm{Se}_{6}$ wire. Interestingly, the top $\mathrm{MoSe}_{2}$ layer can be twisted relative to the bottom layer(s) with the twist angles as high as $5.1^{\circ}$ (Figure $\mathrm{S} 1 \mathrm{~h}$ inset).

Figures S1i show two kinds of edges after selenization: one is zigzag Se edge (left) with a grain boundary like defect embedded next to the edge (marked by white rhombi), the other is zigzag Mo edge (right). Both edges are readily saturated by adsorption of Se atoms. 
(a)

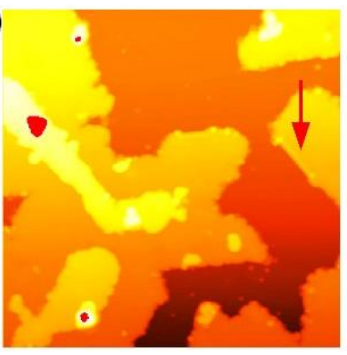

(d)

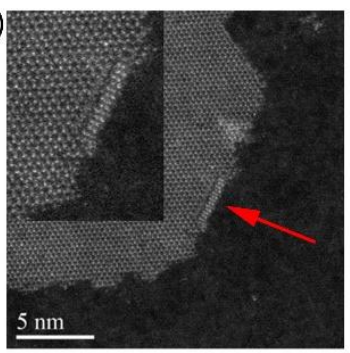

(g)

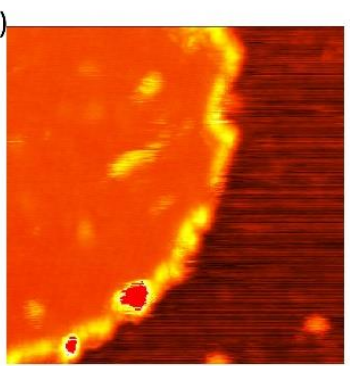

(b)
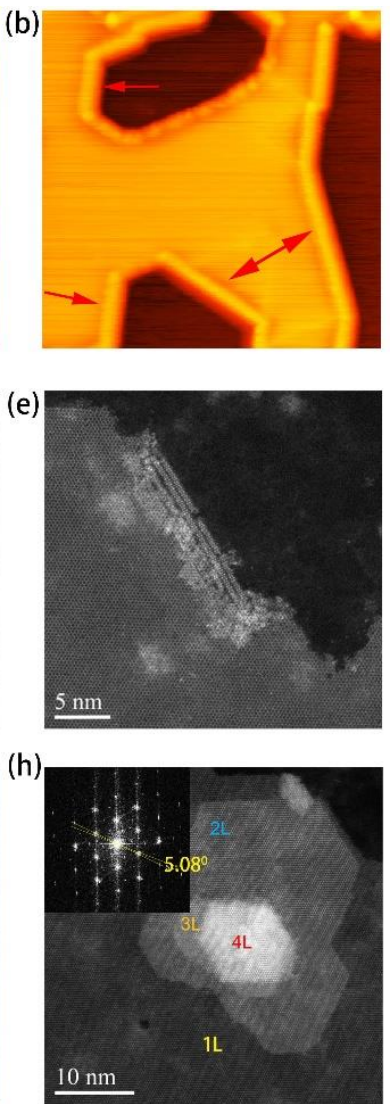
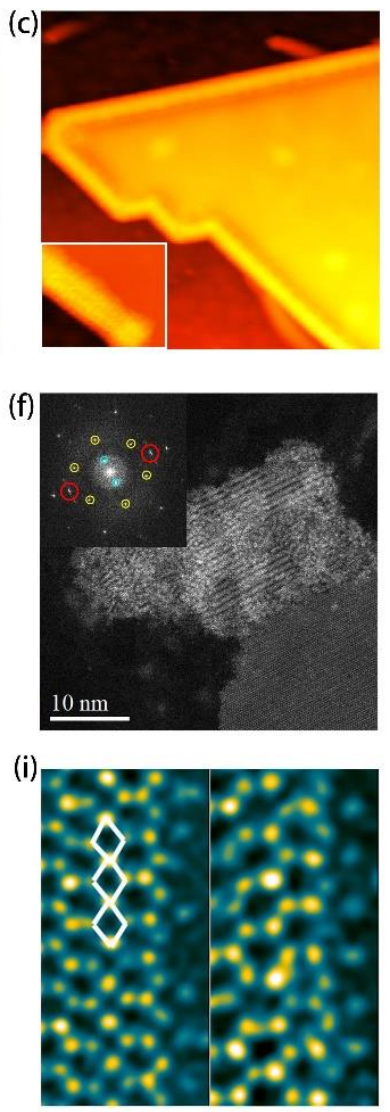

Figure S1. Formation and reversion of $\mathrm{Mo}_{6} \mathrm{Se}_{6}$ nanowires. a-c, STM images showing the gradual formation of $\mathrm{Mo}_{6} \mathrm{Se}_{6}$ nanowires with increasing annealing time from $\sim 1$ to $\sim 30$ minutes (size and sample bias of the images: (a) $100 \times 100 \mathrm{~nm}^{2}, 2.0 \mathrm{~V}$; (b) $50 \times 50 \mathrm{~nm}^{2}, 1.0 \mathrm{~V}$; (c) $30 \times 30 \mathrm{~nm}^{2}, 0.5 \mathrm{~V}$; inset of (c) $7.0 \times 5.8 \mathrm{~nm}^{2}, 0.1$ V). d-f, ADF-STEM images showing single, double and bundled $\mathrm{Mo}_{6} \mathrm{Se}_{6}$ nanowires. Inset of (f): FT of (f) revealing the frequency spectra of $\mathrm{MoSe}_{2}$ (circled yellow) and $\mathrm{Mo}_{6} \mathrm{Se}_{6}$ (circled red for unit cell and blue for inter-wire) lattices. g, STM image $\left(30 \times 30 \mathrm{~nm}^{2}\right.$, sample bias: $\left.-1.0 \mathrm{~V}\right)$ of a $\mathrm{Mo}_{6} \mathrm{Se}_{6}$-containing sample after selenization. $\mathrm{h}$, ADF-STEM image of the $\mathrm{Mo}_{6} \mathrm{Se}_{6}$-containing sample after selenization showing multiple $\mathrm{MoSe}_{2}$ layers. Inset is the FT of the main figure revealing twist of the stacked $\mathrm{MoSe}_{2}$ layers. $\mathrm{i}, \mathrm{ADF}-\mathrm{STEM}$ images of two kinds of edges after selenization. Left: ZZSe-GB4-Se structure (image size: $1.3 \times 2.6 \mathrm{~nm}^{2}$ ); right: ZZMo-Se structure (image size: $1.2 \times 2.4 \mathrm{~nm}^{2}$ ). The white rhombi in the left image mark grain boundary-like defects. In both, the edges are terminated by adsorbed Se atoms (the rightmost row of atoms).

\section{DFT calculations of the edge formation energies as function of chemical potential}

When considering the stabilities of different edge configurations, a ribbon is adopted to simulate a ML$\mathrm{MoSe}_{2}$ terminated by different edges for the DFT calculations. A clean ribbon has two complementary edges: Se-zz and Mo-zz. However, the as cleaved edges are energetically unfavorable, which undergo relaxation and reconstruction. Previous works ${ }^{1-3}$ have suggested the Mo-zz edge is more likely saturated by Se atoms (refer to Figure S2-1a), whereas the Se-zz edge atoms undergo a reconstruction such that every other Se pair (one on 
the top and the other on the bottom layer) forms bond, giving rise to the $2 \times$ periodicity (refer to Figure S2-1c). We adopt these two configurations for the two edges and calculated separately their formation energies $\gamma$ using triangular domains bounded by one of the edges (e.g., Figure S2-2 for the Se-zz case). The formation energy $\left(E_{f}{ }^{\prime}\right)$ of the triangular domain then consists of two parts - the creation of the edges $(\gamma)$ and that of the vertices of the triangle $\left(\varepsilon_{v}\right)$ :

$$
E_{f}^{\prime}=3 \varepsilon_{v}+3 l \gamma
$$

To separate the two contributions, three sizes of triangular domains with changing edge lengths $(l)$ but fixed 3 vertices are considered, thereby the vertex contribution is readily eliminated and the energy $\gamma$ obtained by simply fitting the $E_{f}(l)$ relation.

To calculate the formation energy of $\mathrm{Mo}_{6} \mathrm{Se}_{6}$ attached to either Mo-zz or Se-zz, a $\mathrm{MoSe}_{2}$ ribbon with one edge connected to $\mathrm{Mo}_{6} \mathrm{Se}_{6}$ while the other edge takes the relaxed and reconstructed configuration retaining the $\mathrm{MoSe}_{2}$ stoichiometry is adopted. These are depicted in Figure S2-1b and S2-1d, respectively. The latter represents one that is consistent with the experiment (refer to Figure 2 and the discussions in main text). Another configuration that is also seen by experiment is $\mathrm{Mo}_{6} \mathrm{Se}_{6}$ wires attached to Se-zz edge of $\mathrm{MoSe}_{2}$ by the $4 \mid 4 \mathrm{E}$ interface structure (see Figure $2 \mathrm{e}$ in the main text). Its formation energy is calculated in a similar way as that of Figure S2-1d. Note that for the spectator Mo-edge, the reconstructed configuration adopted in Figure S2-1d is less favorable than that of a Se-saturated edge (cf. Figure S2-1a), but this is irrelevant in deriving the energy of $\mathrm{Mo}_{6} \mathrm{Se}_{6}$ wire, as long as its energy is separately taken into account as derivable by considering again triangular domains of Figure S2-3.

The formation energy $E_{f}$ of the system (ribbon with the Se- and Mo-terminated edges or that with one edge connected to a $\mathrm{Mo}_{6} \mathrm{Se}_{6}$ wire) depends on Se chemical potential $\mu_{\text {Se }}$ according to

$$
E_{f}=E\left(\mathrm{Mo}_{x} \mathrm{Se}_{y}\right)-x E_{0}+(2 x-y) \mu_{\mathrm{Se}}
$$

where $E_{0}$ is the energy of one $\mathrm{MoSe}_{2}$ unit, $x(y)$ is the overall composition of $\mathrm{Mo}(\mathrm{Se})$ atoms in the system, and $(2 x-y)$ represents the 'excess' of Se in the system than is required in forming a stoichiometric $\mathrm{MoSe}_{2}$. The formation energy of the interested edges or $\mathrm{Mo}_{6} \mathrm{Se}_{6}, \gamma$, is then

$$
\gamma=E\left(\mathrm{Mo}_{x} \mathrm{Se}_{y}\right)-x E_{0}+(2 x-y) \mu_{\mathrm{Se}}-\gamma_{s}
$$

with $\gamma_{s}$ being the energy of the 'spectator' edge on the opposite side.

One thing to address is that our MBE-grown $\mathrm{Mo}_{6} \mathrm{Se}_{6}$ nanowire has a rotation angle of $\beta=30^{\circ}$ about the $c$-axis (refer to Figure $2 \mathrm{~b}$ ) instead of $\beta=0^{\circ}$ reported in the literature. ${ }^{4,5}$ Note that the $30^{\circ}$ rotation angle would result in a weaker bonding between $\mathrm{Mo}_{6} \mathrm{Se}_{6}$ and the host $\mathrm{MoSe}_{2}$ (only one of the six Se atoms in $\mathrm{Mo}_{6} \mathrm{Se}_{6}$ is directly linked with the Mo atoms in $\mathrm{MoSe}_{2}$ domains), which would then impact on the properties of the $\mathrm{Mo}_{6} \mathrm{Se}_{6}$ wires. However, in the DFT simulations of freestanding models (without considering the substrate), such rotation angel is not stable 
and would rotate to $\beta=0^{\circ}$ after relaxation. We thus suspect that the experimentally observed rotation angle of $\beta=30^{\circ}$ may be associated with the graphene or HOPG substrates.

(a)

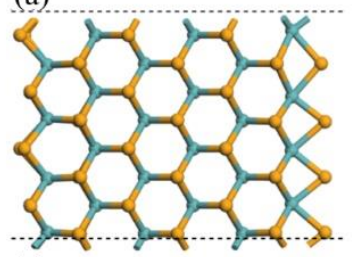

(c)

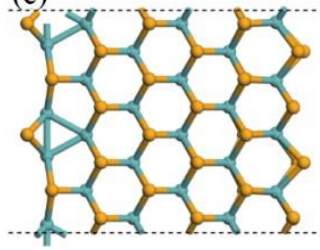

(b)

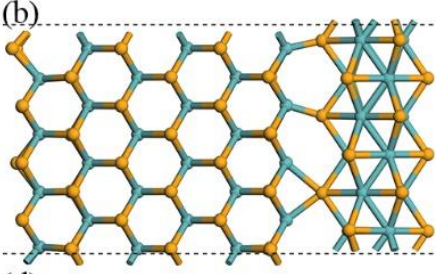

(d)

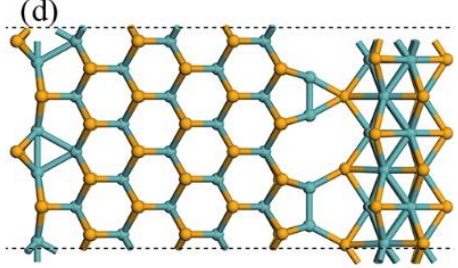

Figure S2-1. Models of ribbons constructed to calculate the formation energies of the edges with or without the $\mathrm{Mo}_{6} \mathrm{Se}_{6}$ wire as functions of Se chemical potential. The yellow balls denote Se atoms and the cyan balls are Mo atoms. (a) and (b) are the optimized Mo edge and a $\mathrm{Mo}_{6} \mathrm{Se}_{6}$ wire attached to Mo-edge, respectively. The 'spectator' edge on the left side of the ribbon is the $\times 2$ reconstructed Se-zz edge. (c) and (d) are optimized Se edge and a $\mathrm{Mo}_{6} \mathrm{Se}_{6}$ attached to the Se-edge, respectively. The 'spectator' edge on the left is a relaxed and reconstructed Mo-zz without changing stoichiometry. Model (d) is identified to be consistent with the experiment shown in Figure 2 in the main text.

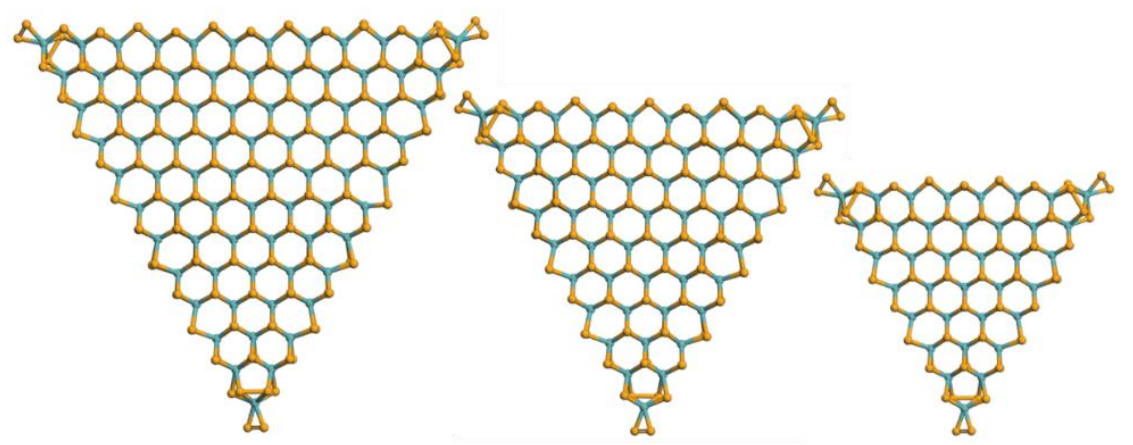

Figure S2-2. Models of triangular domains bounded by the Se-zz edges, which are adopted in calculating the formation energy of the Se terminated edge. 


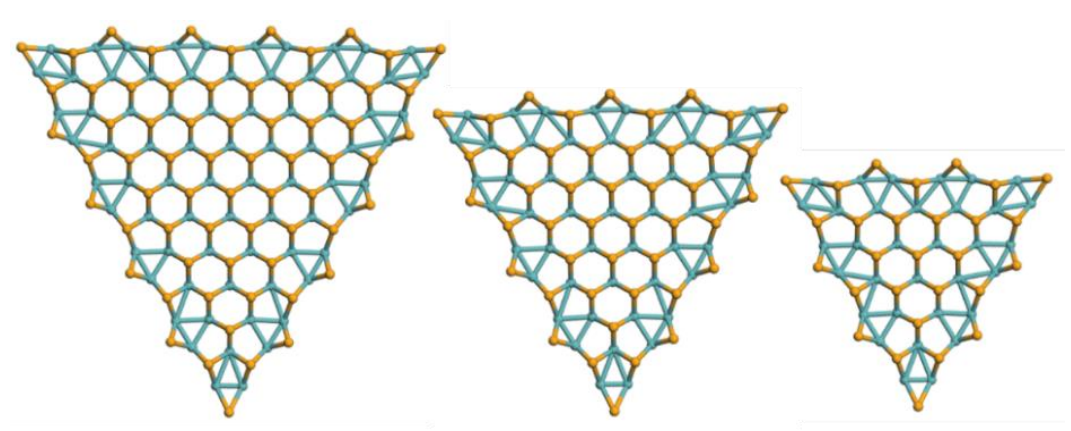

Figure S2-3. Models of triangular domains bounded by the Mo-zz edges adopted in calculating the formation energy of the Mo terminated 'spectator' edge.

\section{Two interface structures connecting $\mathrm{Mo}_{6} \mathrm{Se}_{6}$ and $\mathrm{MoSe}_{2}$}

We found two typical interface structures, i.e., 5|8 and 4|4E, connecting $\mathrm{Mo}_{6} \mathrm{Se}_{6}$ nanowire and $\mathrm{MoSe}_{2} \mathrm{ML}$ sheet. The former is characterized by alternating pentagon-octagon atom rings at the interface (see Figures 3a$3 \mathrm{c}$ in the main text), where $\mathrm{Mo}_{6} \mathrm{Se}_{6}$ wire and $\mathrm{MoSe}_{2}$ sheet stitch together loosely. Figure S3a presents the QSTEM simulated image of the $5 \mid 8$ interface (the $\mathrm{MoSe}_{2}$ domain is on left and $\mathrm{Mo}_{6} \mathrm{Se}_{6}$ nanowire is on right) based on the structure of Figure 3b, which is seen to match well with the experimental STEM result (Figure 3a in main text). Different from the previously reported electron-irradiation-induced $\mathrm{Mo}_{6} \mathrm{Se}_{6}$ nanowires, our MBE-grown $\mathrm{Mo}_{6} \mathrm{Se}_{6}$ nanowire has a rotation angle of $\beta=30^{\circ}$ about the $c$-axis (refer to Figure $3 \mathrm{~b}$ in the main text) instead of $\beta=0^{\circ}$ reported in the literature. ${ }^{4,5}$ The 30 -degree rotation angle would result in a weaker bonding between $\mathrm{Mo}_{6} \mathrm{Se}_{6}$ and $\mathrm{MoSe}_{2}$ (only one of the six Se atoms in $\mathrm{Mo}_{6} \mathrm{Se}_{6}$ is directly linked with the Mo atoms in $\mathrm{MoSe}_{2}$ domains), which would impact on the properties of the $\mathrm{Mo}_{6} \mathrm{Se}_{6}$ wires. For the second, 4|4E, interface structure, it has double 4-atom rings that share the Mo-Se bond at the edge on the $\mathrm{MoSe}_{2} \mathrm{side}_{\text {of }}$ the interface (see Figures 3d-3f in the main text). Figure S3b shows a QSTEM simulated ADF-STEM image of such a model structure, which again agrees with the experimental observation (Figure $3 \mathrm{~d}$ in the main text).

(a)

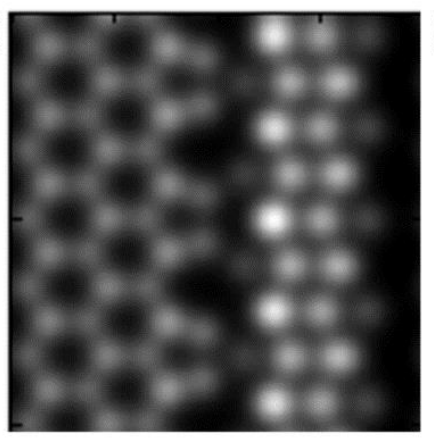

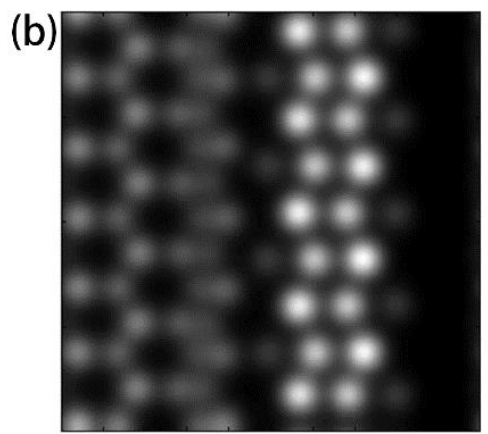

Figure S3. QSTEM simulated images of the two interface structures. a, $5 \mid 8$ interface. b, $4 \mid 4 \mathrm{E}$ interface. The left part showing low contrast in each image are the $\mathrm{MoSe}_{2}$ domains and the right bright region represents the $\mathrm{Mo}_{6} \mathrm{Se}_{6}$ nanowires. 


\section{Phonon spectrum of $\mathrm{Mo}_{6} \mathrm{Se}_{6}$ nanowires}

Under low temperature, some 1D metallic systems may exhibit Peierls instability, which causes periodic lattice distortion and charge density wave (CDW). Phonon spectrum calculated by DFT can be helpful in identifying the existence of such CDW in 1D. Figure S4 shows the calculated phonon spectrum of freestanding $\mathrm{Mo}_{6} \mathrm{Se}_{6}$ nanowires. As seen, there is no imaginary frequency, which indicates the unlikelihood of Peierls-type CDW in the system.

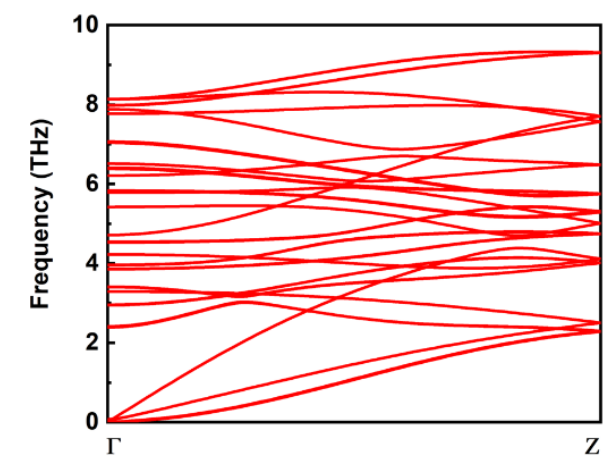

Figure S4. Phonon spectrum of $\mathrm{Mo}_{6} \mathrm{Se}_{6}$ nanowires.

\section{Moiré effect on electronic structure of $\mathrm{Mo}_{6} \mathrm{Se}_{6}$ wires}

For a $\mathrm{MoSe}_{2}$ attached $\mathrm{Mo}_{6} \mathrm{Se}_{6}$ wire as shown in Fig. 3c, a Moiré modulation is present. This will bring an important effect on the electronic structure, such as energy band dispersion relation, of the $\mathrm{Mo}_{6} \mathrm{Se}_{6}$ wire. For example, for a small Moiré potential, an energy gap may be opened at wavevectors corresponding to the $3 a$ periodicity, while the dispersion relation is more or less retained. In the case of strong Moiré potentials, however, the dispersion might be significantly altered or ruined. To evaluate the impact of the Moiré potential in the system, we performed DFT calculations of an oversimplified but manageable model, where an isolated $\mathrm{Mo}_{6} \mathrm{Se}_{6}$ wire is artificially distorted by $\sim 1.65 \times 10^{-2} \mathrm{~nm}$, or $1.25 \%$, for every 3 lattice units along the wire, thus a larger period of $3 a$ is artificially introduced corresponding to the experiment. The calculation result is shown in Fig. S5(a) and S5(b). Comparing to the undistorted wire (Fig. 5c in the main text), there are extra bands, which originate from the band folding effect. Indeed, the $3 a$ super-periodicity of the lattice reduces the Brillouin zone (BZ) size from $2 \pi / a$ to $2 \pi / 3 a$ and the bands are folded into the reduced zone. In particular, the highly dispersive bands close to the original BZ boundary becomes folded into the reduced-BZ close to the boundary $k^{\prime}=\Gamma Z / 3$. Besides, the Moiré potential has led to opening of mini-gaps of the folded bands at the reduced-BZ boundary $k^{\prime}$ (circled in (b)). 

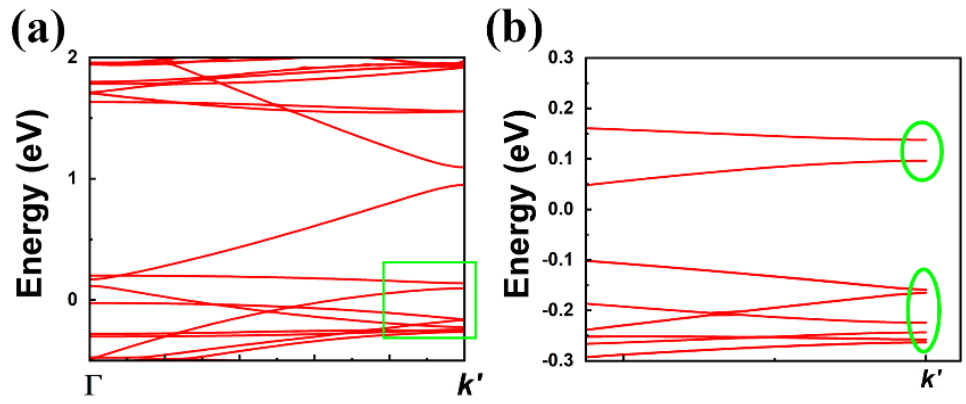

Figure S5. Moiré effect on the band structure of $\mathrm{Mo}_{6} \mathrm{Se}_{6}$ wire, where a super-periodic (periodicity 3a) lattice distortion $\left(\sim 1.65 \times 10^{-2} \mathrm{~nm}\right.$ or $\left.1.25 \%\right)$ is introduced along the wire. (a) DFT calculated band structure in half of the reduced Brillouin zone $\Gamma \rightarrow k^{\prime}$, where $k^{\prime}=\Gamma Z / 3$. (b) Magnified plot of the boxed region of the band, where the circles highlight the folded bands with mini-gaps opened by the Moiré potential.

\section{Experimental methods}

\section{Growth of nanowires}

The growth of $\mathrm{MoSe}_{2}$ thin films was carried out in an MBE chamber having a background pressure of $10^{-10}$ Torr. The flux of molybdenum was generated from an e-beam evaporator operated at the power of $\sim 50 \mathrm{~W}$, and that of selenium was generated from a Knudsen cell held at $\sim 125^{\circ} \mathrm{C}$. HOPG or epitaxial graphene were used as the substrates, where the latter was obtained by annealing $\mathrm{SiC}$ wafer at $\sim 1100^{\circ} \mathrm{C}$ under a $\mathrm{Si}$ flux. The temperature of the substrate was $\sim 500^{\circ} \mathrm{C}$ during $\mathrm{MoSe}_{2}$ growth and the growth rate was $\sim 0.5 \mathrm{MLs} / \mathrm{hr}$ as limited by the flux of Mo $\left(\sim 1.5 \times 10^{11}\right.$ atoms $\left./ \mathrm{cm}^{2} \cdot \mathrm{s}\right)$. Se flux $\left(\sim 1.2 \times 10^{12}\right.$ molecules $\left./ \mathrm{cm}^{2} \cdot \mathrm{s}\right)$ was around 10 times higher than that of Mo. $\mathrm{Mo}_{6} \mathrm{Se}_{6}$ nanowires were obtained by post-growth annealing at temperatures $\geq 800^{\circ} \mathrm{C}$. The length and coverage of the nanowires could be controlled by adjusting the annealing time (normally 1-30 mins). The growing surface was monitored by reflection high-energy electron diffraction (RHEED) operated at $15 \mathrm{keV}$.

\section{STM and STEM Characterizations}

The grown samples were transferred to a standalone Unisoku STM system for the STM/STS measurements at $\sim 5 \mathrm{~K}$ and under the base pressure around $1 \times 10^{-10}$ Torr. Constant current mode of scanning was used with the tunneling current of $100 \mathrm{pA}$. The $\mathrm{dI} / \mathrm{dV}$ spectra were taken by a lock-in amplifier at $1.009 \mathrm{kHz}$. Samples for ADF-STEM characterizations were transferred onto a molybdenum-based TEM grid via micromechanical exfoliation as reported in ref ${ }^{6}$. Atomic-resolution ADF-STEM were performed in a probe-corrected STEM (FEI Titan Chemi STEM) operated at $200 \mathrm{kV}$. The convergence semi-angle was set at $21.4 \mathrm{mrad}$ and the range of acceptance angle of the ADF detector was 53-200 mrad. ADF-STEM image simulations were performed by the QSTEM software with the simulation parameters chosen to be the same as those used experimentally. ${ }^{7}$ 


\section{DFT calculations}

First-principles calculations were carried out using the Vienna ab initio simulation package (VASP), based on the density functional theory (DFT). ${ }^{8}$ The interactions of electrons and the core are treated by projector augmented wave (PAW) method, with the cutoff energy $500 \mathrm{eV} .{ }^{9}$ The Perdew-Burke-Ernzerhof (PBE) formalism of generalized gradient approximation $(\mathrm{GGA})^{10}$ was used for exchange-correlation. The k-point mesh of BZ was set to be $5 \times 1 \times 1$ in the self-consistency cycle. A $5 \times 1 \times 1$ supercell was constructed to obtain the phonon spectrum by using the PHONONPY code with the DFPT method. ${ }^{11}$

\section{REFERENCE}

1. Chen, Y.; Cui, P.; Ren, X.; Zhang, C.; Jin, C.; Zhang, Z.; Shih, C.-K. Nature Communications 2017, 8, (1), 15135 .

2. $\quad$ Helveg, S.; Lauritsen, J. V.; Lægsgaard, E.; Stensgaard, I.; Nørskov, J. K.; Clausen, B. S.; Topsøe, H.; Besenbacher, F. Physical Review Letters 2000, 84, (5), 951-954.

3. Bollinger, M. V.; Lauritsen, J. V.; Jacobsen, K. W.; Nørskov, J. K.; Helveg, S.; Besenbacher, F. Physical Review Letters 2001, 87, (19), 196803.

4. $\quad$ Sang, X.; Li, X.; Zhao, W.; Dong, J.; Rouleau, C. M.; Geohegan, D. B.; Ding, F.; Xiao, K.; Unocic, R. R. Nature communications 2018, 9, (1), 2051.

5. Huang, W.; Wang, X.; Ji, X.; Zhang, Z.; Jin, C. Nano Research 2018, 11, (11), 5849-5857.

6. Hong, J.; Wang, C.; Liu, H.; Ren, X.; Chen, J.; Wang, G.; Jia, J.; Xie, M.; Jin, C.; Ji, W.; Yuan, J.; Zhang, Z. Nano Letters 2017, 17, (11), 6653-6660.

7. Koch, C. T., Determination of core structure periodicity and point defect density along dislocations. 2002.

8. Kresse, G.; Furthmüller, J. Physical review B 1996, 54, (16), 11169.

9. Blöchl, P. E. Physical review B 1994, 50, (24), 17953.

10. $\quad$ Perdew, J. P.; Burke, K.; Ernzerhof, M. Physical review letters 1996, 77, (18), 3865.

11. Togo, A.; Tanaka, I. Scripta Materialia 2015, 108, 1-5. 